\title{
Proposal and Finite-Difference Time-Domain Simulation of Whispering Galley Mode Microgear Cavity
}

\author{
Masayuki Fujita, Student Member, IEEE, and Toshihiko Baba, Member, IEEE
}

\begin{abstract}
The existence of many high $Q$ whispering galley modes in microdisk and microcylinder lasers seriously affects the internal efficiency in lasing operation and disturbs the enhancement of the spontaneous emission factor. To suppress these modes except for one lasing mode, we propose the microgear cavity having a grating with the same period as that of the mode standing wave. The finite-difference time-domain simulation theoretically demonstrates that the microgear selects one resonant mode that satisfies the unique condition regarding the mode order and the phase. This paper describes the dependence of the mode behavior on some structural parameters, and concludes that a deep grating, which seems to be possible in an experiment, allows for sufficient suppression of any types of nonlasing modes and the enhancement of the $Q$ factor of the lasing mode.
\end{abstract}

Index Terms-FDTD, grating, microcavity, microdisk, microgear, spontaneous emission control, whispering gallery mode.

\section{INTRODUCTION}

$\mathbf{S}$ EMICONDUCTOR whispering galley (WG) mode cavities such as microdisks [1]-[6] and microcylinders [7], [8] can be easily miniaturized to a few microns in diameter, while maintaining a high $Q$, owing to total internal reflection at boundaries. As laser diodes, they are expected to have a low threshold or exhibit thresholdless lasing caused by the strong coupling of spontaneous emission into a single lasing mode [2]. In the $1.5-\mu \mathrm{m}$ GaInAsP system, the diameter of photo-pumped and current-injected microdisk lasers have been reduced to 1.6 [3] and $2 \mu \mathrm{m}$ [4], respectively. Room-temperature continuous-wave lasing with a threshold current of $40 \mu \mathrm{A}$ [5] and a spontaneous emission factor (coupling efficiency of spontaneous emission into a lasing mode) of 0.1 [6] have been achieved. However, these devices sometimes exhibit nonlasing modes with high $Q \mathrm{~s}$ in the spontaneous emission spectrum of the active material [9], which increase the threshold current [10] and disturb the enhancement of the spontaneous emission factor [6]. As a nonlasing mode, one may consider the orthogonally-polarized mode. However, this mode can be fully suppressed by employing strained quantum

Manuscript received February 13, 2001; revised June 12, 2001. This work was supported in part by the Ministry of Education, Culture, Sports, Science and Technology, under Grant-in-Aid 13305009, and also by the Japan Society for Promotion of Science under Research for the Future JSPS-RFTF 97P00103 and Research Fellowship 05045.

The authors are with the Faculty of Electrical and Computer Engineering, Yokohama National University, Hodogaya-ku, Yokohama 240-8501, Japan (e-mail: baba@dnj.ynu.ac.jp).

Publisher Item Identifier S 0018-9197(01)08329-4. wells (QWs) as the active material; the transverse magnetic polarization is suppressed by using the electron and heavy-hole transition in compressively strained QWs. As we have used compressively strained QWs in our previous experiments [2], [4]-[6], [8], [11], [12], we limit the following discussion to the transverse electric (TE) polarization. Secondly, one will consider azimuthal and radial modes, which have different numbers of standing waves in these directions. These modes all have different resonant wavelengths with a range of several nanometers to over $10 \mathrm{~nm}$. WG modes are defined as the first-order radial mode. Higher order radial modes having field maxima inside the cavity are automatically suppressed by diffraction losses, when the diameter of the cavity is reduced so as to be comparable to the lasing wavelength. Thus, only different-order azimuthal modes can be nonlasing modes to be managed. Thirdly, one should also consider infinitely degenerate WG modes, which have the same azimuthal mode order and the same resonant wavelength but different phases in the azimuthal direction. Ideally, these modes do not affect lasing characteristics, since all of them equally contribute to the lasing. However, some backscattering of such modes occurs in an imperfect cavity. This causes the coupling between the clockwise and counter-clockwise propagating waves, [13] and breaks the degeneracy between them. The broken degenerate modes still have the same azimuthal order but slightly different resonant wavelengths. Actually, we and other groups observed that some fabricated devices exhibited nonlasing modes with resonant wavelengths less than 1-nm different from that of the lasing mode [5], [6], [11]-[14].

In this paper, we propose and theoretically analyze the $\mathrm{mi}$ crogear cavity that supports one WG mode as the lasing mode and fully suppresses nonlasing modes with different azimuthal order and/or different phase. As illustrated in Fig. 1, it has a grating along the periphery of a circular cavity. By making the grating period to be equal to half the lasing wavelength, degenerate clockwise and counterclockwise waves of the lasing mode are efficiently coupled with each other, and two different modes are formed, each of which has its own phase, resonant wavelength and $Q$ factor. When their $Q$ factors are sufficiently different, stable single-mode lasing is achieved. Regarding a grating formed in a microdisk laser, there is a report describing the directional light extraction by an asymmetric grating [15]. The purpose and the design of our grating are absolutely different. Our grating is rather similar to a conventional linear grating in a distributed feedback (DFB) laser as regards mode selection. However, there are two important differences: 1) our 


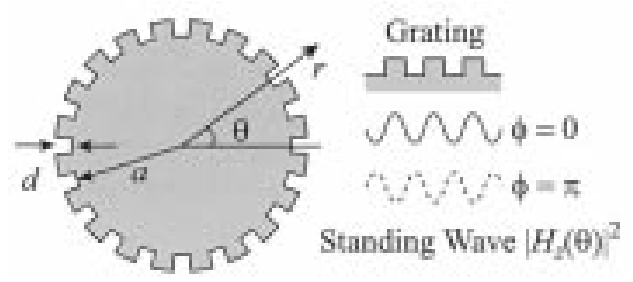

Fig. 1. Schematic of microgear cavity. Phase $\phi=0$ and $\phi=\pi$ are defined such that antinodes of magnetic field locate at convexities and concavities of grating, respectively.

grating can enhance the $Q$ factor of the lasing mode from that obtained by the ideal cavity without grating and 2) it can suppress different phase modes, which cannot be managed by the linear grating in DFB lasers.

In this paper, the influence of nonlasing modes in a microcavity is generally discussed from the rate equation analysis in the next section. Then, a microgear cavity model and the numerical calculation method are explained in Section III. The enhancement of the $Q$ factor is discussed with the suppression of different order azimuthal modes in Section IV and with the suppression of different phase modes in Section V. Finally, the dependence on the grating depth is presented and the optimum design is discussed in Section VI.

\section{INFLUENCE OF NONLASING MODES}

For simplicity, let us consider the situation when there is one nonlasing mode (mode 2) other than the lasing mode (mode 1) in the electron transition spectrum of an active material, and assume that they have the same resonant wavelength $\lambda$ and different $Q$ factors $Q_{1}$ and $Q_{2}$. As mentioned in the previous section, wavelengths for these modes are not the same but slightly different in actual devices, and this is the problem. However, once we decide in this analysis that mode 2 does not contribute to lasing, the same wavelength assumption simulates the worst case of lasing characteristics. Rate equations for the carrier density $N$ and the photon density (photon number divided by the volume of active region) of the $i$ th mode $S_{i}$ are given as

$$
\begin{aligned}
\frac{d N}{d t} & =\frac{P}{V}-G\left(S_{1}+S_{2}\right)-\frac{N}{\tau_{c}} \\
\frac{d S_{i}}{d t} & =G S_{i}-\frac{2 \pi c}{\lambda Q_{i}} S_{i}+\frac{C / 2}{\tau_{r}} N \quad(i=1,2)
\end{aligned}
$$

where

$P \quad$ pump rate;

$G \quad$ net gain coefficient;

$V \quad$ volume of active region;

$\tau_{c} \quad$ carrier lifetime;

$\tau_{r} \quad$ radiative recombination lifetime;

$c \quad$ velocity of light in vacuum.

The nonlinear gain interaction between modes, which occurs in semiconductors by intraband relaxation, is ignored. For this discussion, $C$ is defined as the sum of the spontaneous emission

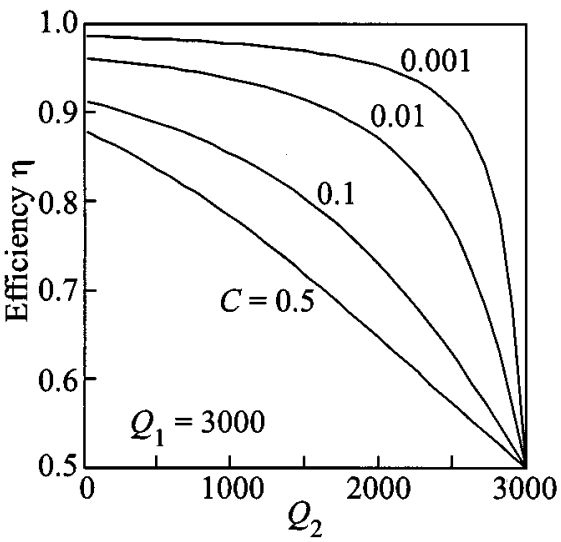

Fig. 2. Efficiency of carriers contributing to lasing mode at threshold calculated with $Q_{2}$ for nonlasing mode.

factor for these modes. The efficiency $\eta$ of the carriers used for the lasing mode at threshold $P=P_{t h}$ is

$$
\eta=\left[\left.\frac{\partial S_{1}}{\partial P}\right|_{P=P_{t h}}\right] /\left[\left.\frac{\partial S_{0}}{\partial P}\right|_{P=P_{t h}}\right]
$$

where $S_{0}$ is the photon density calculated under the condition of pure, single-mode lasing. The threshold gain is independent of the nonlasing mode but only determined by the balance between the gain and the loss for the lasing mode. However, the existence of the nonlasing mode reduces the efficiency $\eta$ and changes lasing characteristics near threshold.

Fig. 2 shows the efficiency $\eta$ calculated with $Q_{2}$ for the nonlasing mode. Here, we modeled a $1.3-\mu \mathrm{m}$-radius GaInAsP microdisk laser with compressively strained QWs of $16 \mathrm{~nm}$ in total thickness [11], $G=g\left(c / n_{g}\right) \ln \left(N / N_{0}\right)$ with the group index of the lasing mode $n_{g}=2.65$, the gain coefficient $g=118 \mathrm{~cm}^{-1}$, and the transparent carrier density $N_{0}=1.5 \times 10^{18} \mathrm{~cm}^{-3}$, $\tau_{c}=1 /\left[B N^{2}+C_{A} N^{3}\right]$ with the radiative recombination coefficient $B=2.5 \times 10^{-10} \mathrm{~cm}^{3} / \mathrm{s}$ and the Auger recombination coefficient $C_{A}=2.5 \times 10^{-29} \mathrm{~cm}^{6} / \mathrm{s}, \tau_{r}=1 / B N^{2}$ and $\lambda=1.55 \mu \mathrm{m}$. It is observed in Fig. 2 that $\eta$ is reduced by a larger $Q_{2}$, and the condition $Q_{2}=Q_{1}$ gives the minimum value $\eta=0.5$. In addition, $\eta$ is reduced by a larger $C$ factor due to the nonclamped carrier density at threshold [6]. In our previous experiment, we estimated $Q_{2} \sim 0.7 Q_{1}$ [11]. Therefore, for $C=0.1$, which was estimated in that experiment, $\eta$ will be reduced to 0.7. As mentioned above, we are not taking account of the nonlinear gain interaction between modes. If we were to do so, further deterioration of the efficiency and an increase in threshold would result. Thus, the nonlasing mode will be a serious problem in microcavity lasers having a large $C$ factor.

\section{Calculation Method And Microgear Model}

For the calculation of modal characteristics in the microgear, we used the 2-D finite-difference time-domain (FDTD) method [16]. First, we assume a circular dielectric as a basic cavity. The radius $a$ is $1.32 \mu \mathrm{m}$ and the refractive index is 2.65 . This index corresponds to the modal index of the TE-polarized mode in the $1.55-\mu \mathrm{m}-$ GaInAsP slab having a thickness of $200 \mathrm{~nm}$ and an index of 3.39. To this structure, we add $N_{g}$ pieces of a comb-like 


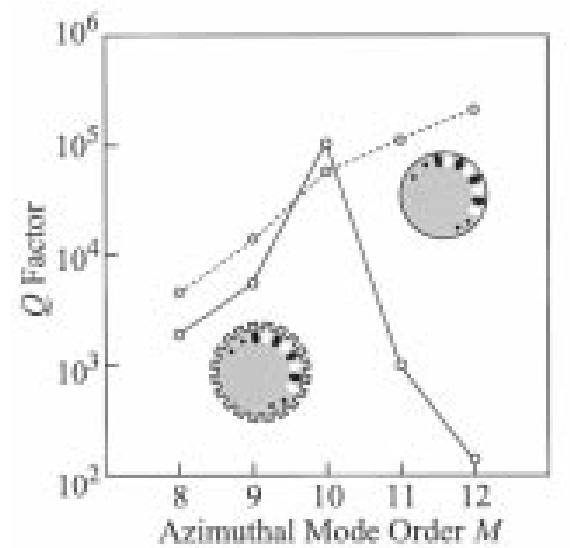

(a)

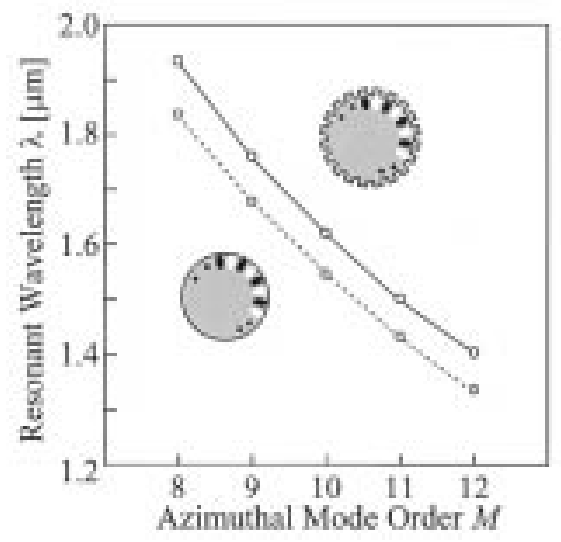

(b)

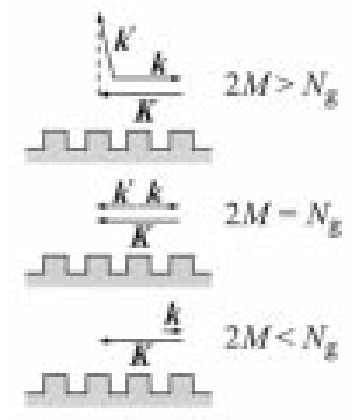

(c)

Fig. 3. (a) $Q$ factor and (b) resonant wavelength of various azimuthal modes. Solid line is for number of grating period $N_{g}=20$ and grating depth $d=$ $195 \mathrm{~nm}$, and dashed line is for basic cavity without grating. (c) The relation of WG mode, diffracted wave and grating, which have wave vectors $\boldsymbol{k}, \boldsymbol{k}^{\prime}$ and $\boldsymbol{K}$, respectively. For grating pitch $\Lambda,|\boldsymbol{K}|=2 \pi / \Lambda$. The conservation of wave vector requires $\boldsymbol{k}+\boldsymbol{K}=\left(\boldsymbol{k}^{\prime} \cdot \boldsymbol{k}\right) \boldsymbol{K} /|\boldsymbol{K}|^{2}$.

grating along the periphery of the cavity to simulate the microgear. Yee's cell is $(15 \mathrm{~nm})^{2}$ square and the time step is $0.03 \mathrm{fs}$. The analytical space is a $(4 a)^{2}$ square. Mur's second-order absorbing condition is used as the boundary condition on peripheries of the analytical space. The initial excitation is given for the magnetic field $H_{z}$ normal to the 2-D plane at four symmetric points in the cavity. The distances from the cavity center to the four points are all $1.04 \mu \mathrm{m}$. The time function is a Gaussian pulse having its peak at a step of 5000 and a spectral broadening of 0.025 times the center angular frequency $\omega_{c}$. The analytical solution of the eigenvalue equation [17] for the modal

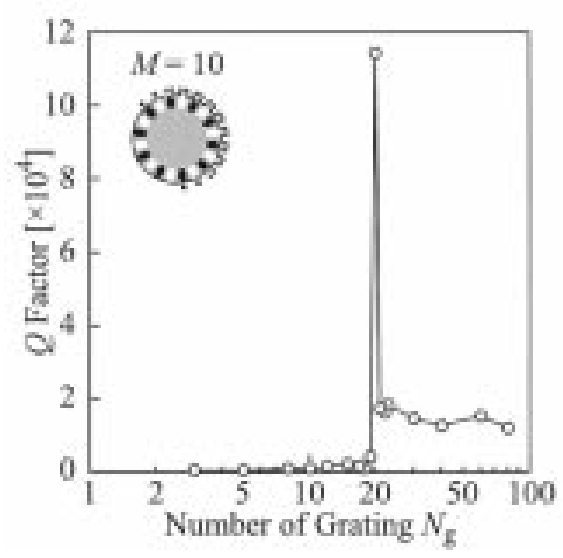

Fig. 4. $Q$ factor versus number of grating period $N_{g}$.

frequency is denoted $\omega_{c}$. The equal amplitude of the excitation is assumed at the four points, but the phase is reversed between neighboring points. As a result, a WG mode with azimuthal mode order $M=10$ and a phase $\phi$ giving antinodes of $H_{z}$ at the four points, can be selectively excited. Also, other modes can be excited by shifting these points suitably from their symmetric positions and adjusting the phase relation. The resonant angular frequency $\omega$ is calculated by the fast Fourier transform of the $2^{16}$ series of $H_{z}$ at an antinode position. The $Q$ factor is calculated from the ratio of the mode energy in the cavity to the time-averaged power radiated outside the cavity at a time-step of over 20000 [16].

When the model is the basic circular cavity, the wavelength $\lambda$ is calculated from the frequency $\omega$ to be $1.547 \mu \mathrm{m}$ and $Q=$ 62100 at $M=10$. Here, the number of the antinode in the standing wave is twice the mode order $M$ (in this case, $2 M=$ 20 ). For the calculation of the microgear, some fixed values are assumed for the grating depth $d$ in Sections IV and V. In Section VI, it is changed to discuss the optimum design of the grating. The number of the grating period $N_{g}$ is changed to investigate its dependence in Section IV, but is fixed to 20 in other sections.

\section{SUPPRESSION OF DIFFERENT ORDER MODES}

Fig. 3(a) and (b) shows the dependence of the $Q$ factor and the resonant wavelength $\lambda$ on the mode order $M$, respectively, where $\phi=0$. Without the grating, $Q$ simply increases with an increase in $M$ due to a shortening of $\lambda$ and the resulting lower diffraction loss. With the grating, $Q$ for $M=10\left(2 M=N_{g}\right)$ is slightly larger than that without the grating. This mode order satisfies the Bragg condition that the wavenumber $|\boldsymbol{K}|$ of the grating is twice the wavenumber $|k|$ of the mode. Under this condition, the clockwise WG mode is coupled to the counterclockwise WG mode. This increase in $Q$ factor is a very interesting phenomenon in the microgear. The reason for this will be explained in Section V. Except for this case, $Q$ is much smaller than that without the grating. Furthermore, it is seen that $Q$ for $2 M>N_{g}$ is smaller than that for $2 M<N_{g}$. The reason for this can be explained such that, for $2 M>N_{g}$, the Bragg diffracted wave couples with radiation modes outside the cavity so as to conserve the wave vector. On the other hand, 

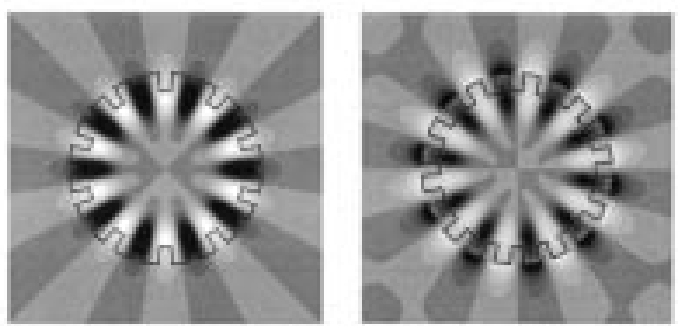

(a)
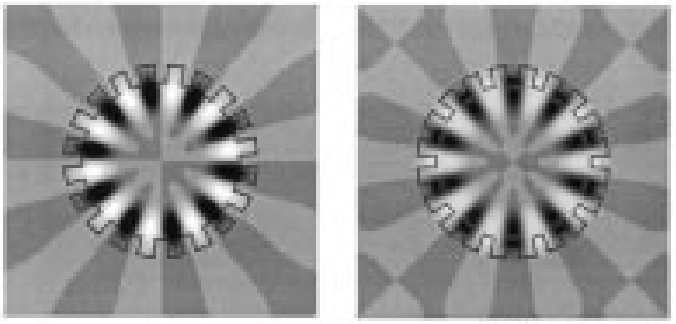

(b)
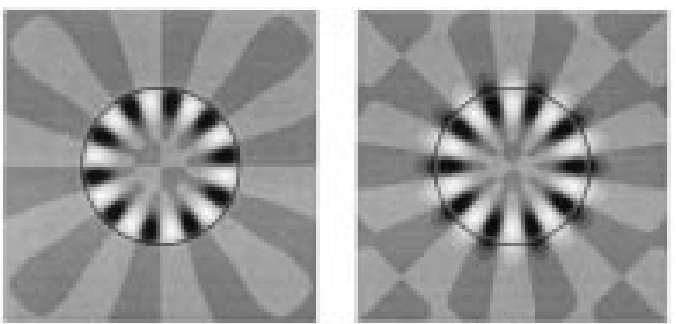

(c)
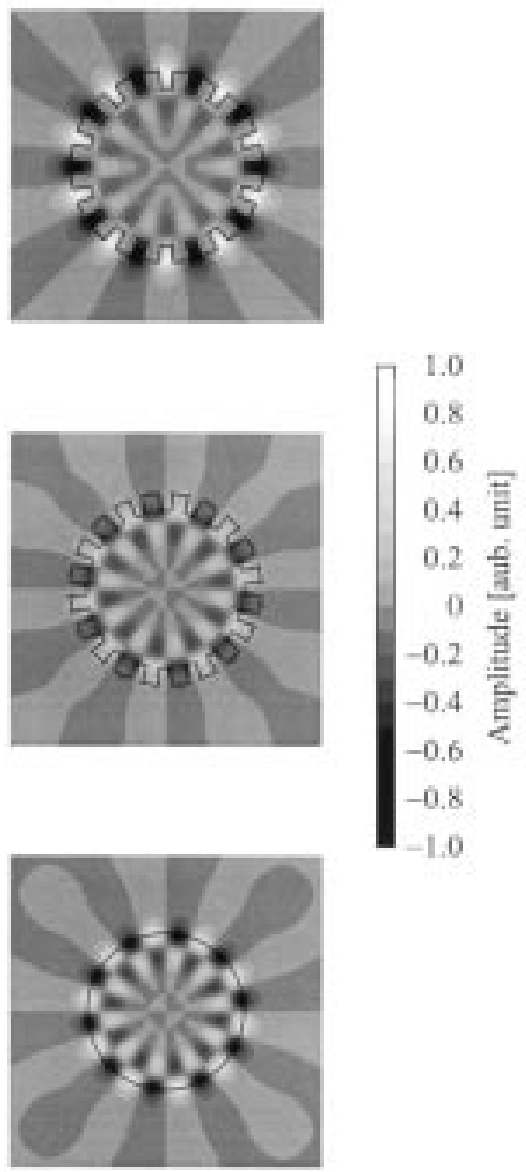

Fig. 5. Simulated field profiles for grating depth $d=300 \mathrm{~nm}$ and number of grating period $N_{g}=20$, and those without grating. (a)-(c) Mode order $M$ of $\phi=\pi$ mode, $\phi=0$ mode, and mode without grating, respectively. Left: normal component of magnetic field $H_{z}$. Middle: radial component of electric field $E_{r}$. Right: angular component of electric field $E_{\theta}$.

for $2 M<N_{g}$, there are no waves that simply satisfy this condition, as illustrated in Fig. 3(c). The wavelength $\lambda$ with the grating is always longer than that without grating, since the effective orbital length is increased by the grating. Fig. 4 shows the $Q$ factor at $M=10$ calculated with number $N_{g}$. Only when $N_{g}=2 M=20$ is $Q$ larger than that without the grating. This result and the difference between for $N_{g}>2 M$ and for $N_{g}<2 M$ can be explained similarly as for Fig. 3 .

\section{Suppression of Different Phase Mode}

A DFB laser with a shallow grating buried by a semiconductor generally operates by either of two modes beside the stopband, which have the same phase near the ends of the cavity and different phases around the center of the cavity. In the microgear, there are two modes with entirely different phases. However, one of these modes can be sufficiently suppressed. This arises from large differences of the field profile and the $Q$ factor between these modes, which come from the large index difference at boundaries. Fig. 5 shows examples of the field profile with and without grating. For those with the grating, the excitation points are moved so that $\phi=0$ or $\pi$. In general, phases of $H_{z}$ and $E_{r}$ are shifted by $\pi / 2$, and phases of $H_{z}$ and $E_{\theta}$ are the same, since the former two contribute to the mode energy in the cavity and the latter two contribute to the radiated power to be the mode loss. A larger difference in the field profile between $H_{z}$ and $E_{r}$ excites a larger $E_{\theta}$, and results in larger mode loss and lower $Q$. For the assumed polarization, $E_{r}$ is discontinuous at round boundaries of the cavities, and largely spreads to free space. As shown in Fig. 5(a), $E_{r}$ of the $\phi=\pi$ mode, whose antinodes are located on convexeties of the grating, is largely spreading, compared with $H_{z}$. On the other hand, as shown in Fig. 5(b), the spreading of $E_{r}$ of the $\phi=0$ mode, whose antinodes are located on concavities of the grating, is properly suppressed. Thus, the $\phi=\pi$ mode excites a larger radiated power than the $\phi=0$ mode. However, $Q_{\pi}=3000$ and $Q_{0}=87500$ for the $\phi=\pi$ and $\phi=0$ modes, respectively, and such very small $Q_{\pi}$ cannot be explained only by the above discussion. Another important point is the positions of the antinodes of $E_{\theta}$ for the $\phi=\pi$ mode; they are outside the concavities of the grating. This accelerates the radiation loss, and reduces $Q_{\pi}$ to a value much lower than $Q_{0}$. Note that the difference between $E_{r}$ and $H_{z}$ of the $\phi=0$ mode is also smaller than that without grating. This is the reason that $Q_{0}$ is larger than that without the grating.

One may think that a large $Q$ is also obtained by a large cavity without the grating. However, this discussion is not so attractive. For example, let us consider a slightly larger cavity with a radius $a=1.39 \mu \mathrm{m}$ (originally $1.32 \mu \mathrm{m}$ ). Even for this, $Q$ determined 


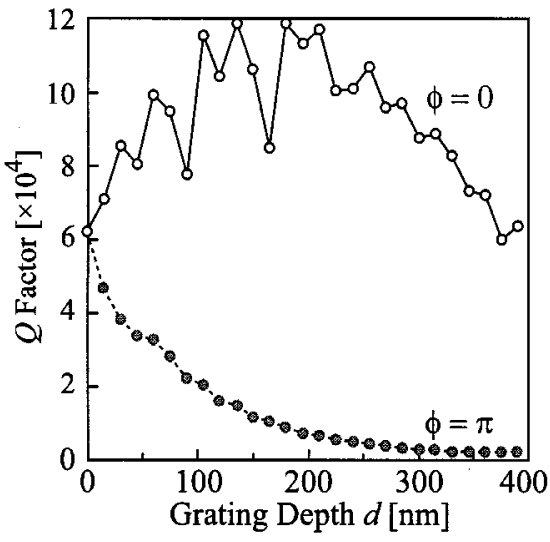

(a)

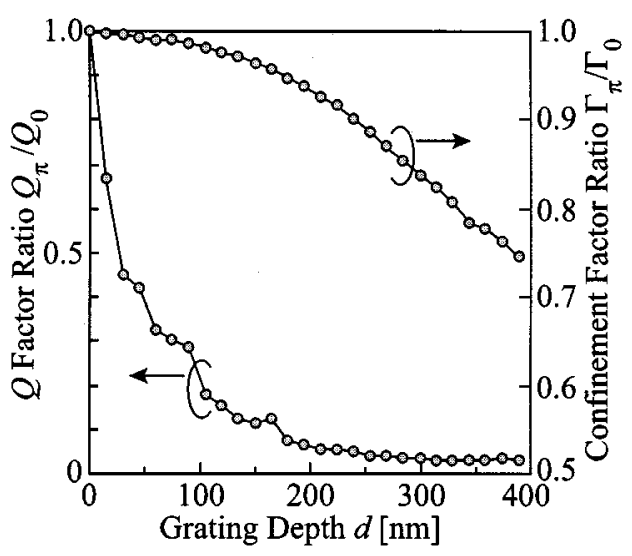

(b)

Fig. 6. Dependence of modes on grating depth $d . M$ and $N_{g}$ are 10 and 20, respectively. (a) Shows $Q$ factor versus grating depth $d$. (b) Shows ratio $Q_{\pi} / Q_{0}$ and $\Gamma_{\pi} / \Gamma_{0}$ with $d$.

by the diffraction loss does not change, since the resonant wavelength $\lambda$ is almost proportional to the radius. If the radius is increased to a value $10 \%$ larger than the original one, the order of the WG mode at $\lambda \sim 1.55 \mu \mathrm{m}$ is changed to $M=11$ and $Q$ is increased. Here, it should be noted that lasing always occurs by even order azimuthal modes in actual microdisk lasers so that the scattering loss of the mode at the edge of the posts, which support the microdisk and have a square cross-section, is minimized [12]. Therefore, an increase in radius of over $20 \%$ is required to change the mode order to $M=12$ for a larger $Q$ factor. However, such a large increase in radius causes a high threshold current. Thus, we can say that the enhancement of $Q_{0}$ without changing the radius and the azimuthal order is a unique advantage of the microgear.

The confinement factor $\Gamma$ is evaluated as the ratio of the mode energy in the cavity to that in the analytical space. Corresponding to the previous discussion, $\Gamma_{\pi}=0.80$, while $\Gamma_{0}=0.96$. Due to the difference in $Q$ and in $\Gamma$, the $\phi=0$ mode will be selected as the lasing mode and the $\phi=\pi$ mode the nonlasing mode. Since the effective orbital length of the mode is also changed by the different field spreading, the resonant wavelength $\lambda_{0}$ is $1.628 \mu \mathrm{m}$ and $\lambda_{\pi}$ is $1.684 \mu \mathrm{m}$. In this case, the mode spacing is as large as $56 \mathrm{~nm}$.

\section{DEPENDENCE ON GRATING DEPTH}

Fig. 6(a) shows the dependence of the $Q$ factor on the grating depth $d$. We use the word "depth" as is usually used for gratings, although our model assumes projections added to the basic cavity. $Q_{0}$ is increased and decreased for $d<200 \mathrm{~nm}$ and for $d>200 \mathrm{~nm}$, respectively. However, it is always larger than that without the grating for the same reason, as explained in Section V. The decrease is considered to be due to the large spreading of the mode fields outside the cavity and the concomitant increase in diffraction loss. $Q_{\pi}$ simply decreases with $d$, since both fields are spreading to outside the cavity. Fig. 6(b) shows the dependence of the ratio $Q_{\pi} / Q_{0}$ and $\Gamma_{\pi} / \Gamma_{0}$ on the depth $d$. For $Q_{\pi} / Q_{0}<0.1$, which ensures that the efficiency $\eta>0.9$ and $C=0.1$, requires that $d>180 \mathrm{~nm}$. However, this requirement will be relieved by considering the confinement factor $\Gamma_{\pi}$ to be lower than $\Gamma_{0}$.

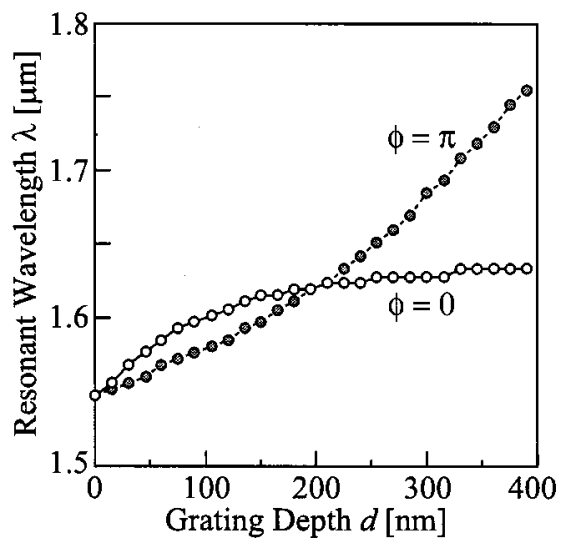

Fig. 7. Wavelength shift versus grating depth $d . M$ and $N_{g}$ are 10 and 20, respectively.

Fig. 7 shows the dependence of the resonant wavelength $\lambda$ on the depth $d$. For the $\phi=0$ mode, $\lambda_{0}$ rapidly increases and saturates for $d<180 \mathrm{~nm}$ and for $d>180 \mathrm{~nm}$, respectively. This can also be explained by the behavior of the effective orbital length and the optical confinement. For a shallow grating, the orbital length is expanded, while strong optical confinement is maintained at the grating and the effective modal index is rather increased. These lead to a longer $\lambda_{0}$. For a deeper grating, the confinement is weakened and the effective index is decreased, and this compensates for the effect of the long orbital length. Thus, the change of $\lambda_{0}$ is saturated. On the other hand, $\lambda_{\pi}$ for the $\phi=\pi$ mode exhibits a simple increase, since the expansion of the orbital length is much faster than the decrease in optical confinement and effective modal index.

For $d>300 \mathrm{~nm}$, the space between $\lambda_{0}$ and $\lambda_{\pi}$ is over $50 \mathrm{~nm}$. As shown in [6], the $C$ factor of a mode detuned from the spontaneous emission peak to a longer wavelength decays exponentially. If the space is larger than $50 \mathrm{~nm}$, the $C$ factor of the nonlasing mode will be one-fourth or smaller than the maximum value. The material gain for such a longer wavelength nonlasing mode will also be smaller. These effects enhance the suppression of the spontaneous and stimulated emission of the nonlasing mode in the microgear. 
By the way, one may be concerned about the increase in surface recombination at the complex edge profile of the microgear, when the grating is deep. Considering the sidewall surface of the grating, surface recombination in the microgear with $d=$ $300 \mathrm{~nm}$ is estimated to be twice that for the basic cavity. Still, this is not serious for the $1.55-\mu \mathrm{m}$ GaInAsP system because of its low surface recombination velocity $\left(\sim 1 \times 10^{4} \mathrm{~cm} / \mathrm{s}\right)$. The rate equation analysis [11] indicates a $30 \%$ increase in threshold due to this effect. However, this increase is fully compensated for by the enhancement of the $Q$ factor in the microgear. In addition, we have reported the effective reduction in surface recombination of GaInAsP by the $\mathrm{CH}_{4}$ plasma irradiation [19]. We expect this method to solve the problem of the microgear.

\section{CONCLUSION}

Using a microgear cavity having a grating that matches the standing wave of the WG mode, the spontaneous and stimulated emission associated with different orders and phase modes are suppressed, and stable single-mode operation is expected. In addition, the $Q$ factor of the lasing mode is enhanced by the well-balanced confinement of the electric and magnetic fields of the WG mode. A deeper grating suppresses the different phase modes more efficiently.

Besides these, we expect this structure to solve another more realistic problem, i.e., the random roughness at cavity sidewalls, which is caused during the fabrication process. It will be reduced by intentionally introducing corrugated patterns of the microgear. This is also very effective for improving the laser performance, since sidewall roughness is one of the crucial factors that determine the cavity loss in these devices [11].

\section{ACKNOWLEDGMENT}

The authors would like to thank Prof. Y. Kokubun of Yokohama National University, and Prof. K. Iga, Prof. S. Arai, Prof. F. Koyama, and Associate Prof. T. Miyamoto of Tokyo Institute of Technology for encouragement and helpful suggestions.

\section{REFERENCES}

[1] S. L. McCall, A. F. J. Levi, R. E. Slusher, S. J. Pearton, and R. A. Logan, "Whispering gallery mode microdisk lasers," Appl. Phys. Lett., vol. 60, pp. 289-291, 1992.

[2] T. Baba, "Photonic crystals and microdisk cavities based on GaInAsP-InP system," IEEE J. Select. Topics Quantum Electron., vol. 3, pp. 808-830, 1997.

[3] A. F. J. Levi, S. L. McCall, S. J. Pearton, and R. A. Logan, "Room temperature operation of submicrometer radius disc laser," Electron. Lett., vol. 29, pp. 1666-1667, 1993.

[4] T. Baba, M. Fujita, A. Sakai, M. Kihara, and R. Watanabe, "Lasing characteristics of GaInAsP/InP strained quantum-well microdisk injection lasers with diameter of 2-10 $\mu \mathrm{m}$," IEEE Photon. Technol. Lett., vol. 9, pp. 878-880, 1997.

[5] M. Fujita, R. Ushigome, and T. Baba, "Continuous wave lasing in GaInAsP microdisk laser with threshold of $40 \mu \mathrm{A}$," Electron. Lett., vol. 27, pp. 790-791, 2000.

[6] -, "Large spontaneous emission factor of 0.1 in a microdisk injection laser," IEEE Photon. Technol. Lett., vol. 13, pp. 403-405, 2001.

[7] A. F. J. Levi, R. E. Slusher, S. L. McCall, S. J. Pearton, R. A. Logan, and W. S. Hobson, "Room-temperature lasing action in $\mathrm{In}_{0.51} \mathrm{Ga}_{0.49} \mathrm{As} / \mathrm{In}_{0.2} \mathrm{Ga}_{0.8}$ As microcylinder laser diode," Appl. Phys. Lett., vol. 62, pp. 2021-2023, 1993.
[8] M. Fujita, T. Baba, A. Matsutani, F. Koyama, and K. Iga, "A novel GaInAsP microcylinder laser with $\mathrm{AlInAs}\left(\mathrm{O}_{x}\right)$ claddings," in Proc. Int. Topical Meet. Indium Phosphide and Related Materials, Davos, Switzerland, 1999, TuB1-5.

[9] N. C. Frateschi and A. F. J. Levi, "Resonant modes and laser spectrum of microdisk lasers," Appl. Phys. Lett., vol. 66, pp. 2932-2934, 1995.

[10] S. A. Backes, J. R. A. Cleaver, A. P. Heberle, J. J. Baumberg, and K. Köhler, "Threshold reduction in pierced microdisk lasers," Appl. Phys. Lett., vol. 74, pp. 176-178, 1999.

[11] M. Fujita, A. Sakai, and T. Baba, "Ultrasmall and ultralow threshold GaInAsP-InP microdisk injection lasers: Design, fabrication, lasing characteristics, and spontaneous emission factor," IEEE J. Select. Topics Quantum Electron., vol. 5, pp. 673-681, 1999.

[12] M. Fujita, R. Ushigome, and T. Baba, " $40-\mu$ A-threshold lasing in GaInAsP microdisk lasers," in Tech. Digest Optoelectronics and Commun. Conf., Makuhari, 2000, 14C3-5.

[13] D. S. Weiss, V. Sandoghdar, J. Hare, V. Lefèvre-Seguin, J. M. Raimond, and S. Haroche, "Splitting of high- $Q$ Mie modes induced by light backscattering in silica microspheres," Opt. Lett., vol. 20, pp. 1835-1837, Sept. 1995.

[14] D. S. Song, J. K. Hwang, C. K. Kim, I. Y. Han, D. H. Jang, and Y. H. Lee, "InGaAsP microdisk lasers on $\mathrm{Al}_{x} \mathrm{O}_{y}$," IEEE Photon. Technol. Lett., vol. 12, pp. 954-956, 2000.

[15] A. F. J. Levi, R. E. Slusher, S. L. McCall, J. L. Glass, S. J. Pearton, and R. A. Logan, "Directional light coupling from microdisk lasers," Appl. Phys. Lett., vol. 62, pp. 561-563, 1993.

[16] A. Sakai and T. Baba, "FDTD simulation of photonic devices and circuits based on circular and fan-shaped microdisks," J. Lightwave Technol., vol. 17, pp. 1493-1499, 1998.

[17] J. A. Stratton, Electro-Magnetic Theory. New York: McGraw-Hill, 1941, ch. 6.

[18] H. Ichikawa, K. Inoshita, and T. Baba, "Reduction in surface recombination of GaInAsP microcolumns by $\mathrm{CH}_{4}$ plasma irradiation," Appl. Phys. Lett., vol. 68, pp. 2119-2121, 2001.

Masayuki Fujita (S"00) was born in Yokohama City, Japan, in 1974. He received the B.E. and M.E. degrees in 1997 and 1999, respectively, from the Division of Electrical and Computer Engineering, Yokohama National University, Yokohama, Japan, where he is currently working toward the Ph.D. degree and studying spontaneous emission control in ultrasmall disk lasers.

$\mathrm{He}$ achieved the first room-temperature continuous-wave operation of microdisk lasers in 1998, the lowest threshold current of $40 \mu \mathrm{A}$ in GaInAsP lasers, and the largest spontaneous emission factor of 0.1 .

Mr. Fujita is a member of the Japan Society of Applied Physics (JSAP). He received the Best Student Paper Award from the Optoelectronic and Communication Conference in 2000, and the Academic Encouragement Award from JSAP in 2001.

Toshihiko Baba (M'93) was born in Nagano Prefecture, Japan, in 1962. He received the B.E., M.E., and Ph.D. degrees from the Division of Electrical and Computer Engineering, Yokohama National University, Yokohama, Japan, in 1985, 1987, and 1990, respectively.

During his Ph.D. work, he was engaged in antiresonant reflecting optical waveguides (ARROWs) and integrated lightwave circuits. In 1990, he joined the Precision and Intelligence Laboratory, Tokyo Institute of Technology, as a Research Associate. In 1991, he first calculated the spontaneous emission factor in microcavity VCSELs. In 1993, he achieved the first room-temperature continuous-wave operation of a long wavelength VCSEL. In 1994, he became an Associate Professor of Yokohama National University. From 1996 to 1997, he reported the lasing of a microdisk injection laser with the smallest cavity, the proposal and the first lasing of a deep-grating Bragg reflector, and fabrication and the first observation of light emission characteristics of photonic crystals. In 1999, he succeeded in demonstrating a photonic crystal waveguide operating at lightwave frequencies. His current research is the spontaneous emission control in microdisk lasers and various device applications based on semiconductor photonic crystals.

Dr. Baba is a member of the Institute of Electronics, Information and Communication Engineers (IEICE), the Japan Society of Applied Physics, and the American Physics Society. He received the Niwa Memorial Prize in 1991, the Best Paper Award of the Micro-Optic Conference in 1993 and 1999, a Paper Award and Academic Encouragement Award from IEICE in 1994, and Marubun Research Encouragement Award in 2000. 